\title{
HMGA2: A biomarker significantly overexpressed in high-grade ovarian serous carcinoma
}

\author{
Aparna Mahajan ${ }^{1}$, Zhaojian Liu ${ }^{1}$, Lan Gellert ${ }^{2}$, Xuanyi Zou², Guangyu Yang ${ }^{1,3}$, Peng Lee ${ }^{2}$, \\ Ximing Yang ${ }^{1,3}$ and Jian-Jun Wei ${ }^{1,3}$ \\ ${ }^{1}$ Department of Pathology, Northwestern University, Feinberg School of Medicine, Chicago, IL, USA; \\ ${ }^{2}$ Department of Pathology, New York University School of Medicine, New York, NY, USA and ${ }^{3}$ Robert H. Lurie \\ Comprehensive Cancer Center of Northwestern University, Chicago, IL, USA
}

\begin{abstract}
Ovarian carcinoma consists of a group of histologically heterogeneous diseases involving distinct tumorigenic pathways. High-grade papillary serous carcinoma of the ovary is commonly associated with p53 mutations. HMGA2, an oncofetal protein, is found to be overexpressed in ovarian cancer. To study the function of HMGA2 in ovarian cancer, it is important to know which subtypes of ovarian cancer are associated with HMGA2 overexpression. In this study, we collected six different types of ovarian cancer and examined HMGA2 expression by immunohistochemistry, along with HMGA1, p53 and Ki-67. We found that HMGA2 overexpression was significantly higher in high-grade papillary serous carcinoma $(64 \%)$ and carcinosarcoma $(60 \%)$ than in other types of ovarian cancers (7-23\%). HMGA2 overexpression was moderately associated with dominant $p 53$ mutations $(R=0.51)$. In addition, the microRNA in situ analysis revealed that let-7b, the HMGA2-negative regulators, were significantly lost in high-grade serous carcinoma. Our findings suggest that HMGA2 is an important molecular change significantly related to high-grade papillary serous carcinoma and is less common in other histological types of ovarian cancer.

Modern Pathology (2010) 23, 673-681; doi:10.1038/modpathol.2010.49; published online 12 March 2010
\end{abstract}

Keywords: ovarian carcinoma; papillary serous carcinoma; HMGA2; p53; immunohistochemistry

Epithelial ovarian cancer accounts for about 3\% of total cancer cases in women. However, ovarian cancer is one of the most lethal gynecological malignancies. An estimated 21,550 new cases were expected in the United States in 2008. An estimated 15,520 deaths were expected in 2008. ${ }^{1}$ Ovarian cancer is a group of heterogeneous diseases and consists of different histological types, which can be readily differentiated by histological evaluation. Genome-wide global gene analysis further defines distinct expression profiles of different types of ovarian cancer. ${ }^{2}$ Different histological types of ovarian cancer seem to be regulated by different pathogenic pathways. ${ }^{3}$

HMGA2, a high-mobility-group AT-hook (HMGA) protein, is a nonhistone DNA-binding factor and binds to AT-rich sequences in the minor groove of

Correspondence: Dr J-J Wei, MD, Department of Pathology, Northwestern University, SOM, Feinberg 7-334, 251 East Huron Street, Chicago, IL 60611, USA.

E-mail: jianjun-wei@northwestern.edu

Received 21 September 2009; revised 13 November 2009; accepted 7 December 2009; published online 12 March 2010 the DNA helix. HMGA2 is expressed in embryonic tissue, but not in most adult tissues, ${ }^{4,5}$ and is an important regulator for cell growth, differentiation, apoptosis and malignant transformation. ${ }^{6}$

HMGA2 is overexpressed in many malignant neoplasms,${ }^{6}$ including ovarian cancer. ${ }^{7-10}$ In animal models, overexpression of HMGA2 is found to be an early event in ovarian tumorigenesis. ${ }^{7,9}$ Studies have shown that silencing of HMGA2 gene expression prevents $R A S$-induced transformation of thyroid cells, ${ }^{11}$ inhibits ovarian cancer growth in nude mice ${ }^{7}$ and results in growth inhibition and increased apoptosis of liposarcoma cells. ${ }^{12}$ HMGA2 may be an important tumorigenic factor associated with cancer development. The expression of HMGA2 is regulated by microRNA. Studies show that let-7s can specifically repress HMGA2 expression both in vivo and in vitro, revealing the regulatory role of let7 in HMGA2 expression. ${ }^{9,13-17}$ Downregulation of let-7s is common in ovarian cancer. ${ }^{16,18}$

To understand the function of HMGA2 in human ovarian cancer, it is important to characterize which type of ovarian cancer is associated with HMGA2 
overexpression. In this study, we examined HMGA2 expression along with $H M G A 1, p 53$ and $\mathrm{Ki}-67$ in various types of ovarian cancer. Our results show that $H M G A 2$ overexpression was significantly higher in high-grade papillary serous carcinoma than in other types of ovarian cancer. In addition, we found that let-7b, the negative regulator of $H M G A 2$, was significantly downregulated in high-grade papillary serous carcinoma. We suggest that HMGA2 can be one of the important molecules in the tumorigenesis of high-grade papillary serous carcinoma.

\section{Materials and methods}

\section{Case Selection}

The cases were collected after surgery at Northwestern Memorial Hospital and New York University from 1996 to 2009. Approval of the institutional research board from both institutions was obtained. A total of 115 cases of ovarian cancer were collected for the study. This included histological diagnosis of high-grade papillary serous carcinoma $(N=30)$, serous borderline tumor $(N=10)$, malignant mixed Mullerian tumor (carcinosarcoma, $N=15$ ), endometrioid ovarian carcinoma $(N=30)$, mucinous ovarian carcinoma $(N=15)$ and clear cell ovarian carcinoma $(N=15)$. Patients' ages at surgery, tumor sizes, FIGO grades and stages and lymph node metastasis are summarized in Table 1.

\section{Tissue Preparation, Antibodies and Immunohistochemistry}

All cases were reviewed by two pathologists. Tissue cores were collected from tumor and control sections of each case (normal fallopian tubes for serous carcinoma, endometrium for endometrioid carcinoma) for tissue microarray. Antibodies used for this study included HMGA1 (kindly provided by Dr E Hernando), HMGA2 (BioCheck Inc., Foster City, CA, USA), Ki-67 (cell proliferation marker; Neomarkers, Fremont, CA, USA) and p53 (Dako, Carpinteria, CA, USA). Tissue microarrays were sectioned $4 \mu \mathrm{m}$ in thickness. After deparaffinization and antigen retrieval, all immunohistochemical staining was performed on a Ventana Nexus automated system (Tucson, AZ, USA). In brief, endogenous peroxidase activity was blocked with $3 \%$ hydrogen peroxide. Primary antibodies were detected using standard biotinylated anti-mouse or anti-rabbit secondary antibodies.

\section{let-7b and let-7c microRNA In Situ Hybridization}

The hybridization system and probes of miRCURY LNA, including let-7b, let-7c and U6, were purchased from Exiqon (Vedbaek, Denmark). The detailed procedure for in situ hybridization was followed as per manufacturer's protocol. ${ }^{19}$ In brief, $4 \mu \mathrm{m}$ tissue microarray slides were prepared. After deparaffinization and deproteinization, the slides
Table 1 Clinical summary of the different types of ovarian carcinoma in this study

\begin{tabular}{|c|c|c|c|c|c|c|}
\hline & $H G-P S C$ & $S B T$ & $M M M T$ & $E O C$ & $M O C$ & CCOC \\
\hline \multicolumn{7}{|l|}{ Age } \\
\hline Average & 66 & 47 & 67 & 55 & 46 & 57 \\
\hline Range & $40-93$ & $28-73$ & $51-88$ & $36-71$ & $18-93$ & $34-85$ \\
\hline \multicolumn{7}{|c|}{ Tumor size $(\mathrm{cm})$} \\
\hline$<=5$ & 6 & 4 & 6 & 2 & 0 & 5 \\
\hline $5.1-20$ & 28 & 5 & 8 & 7 & 7 & 8 \\
\hline$>20$ & 0 & 1 & 0 & 1 & 8 & 0 \\
\hline \multicolumn{7}{|l|}{ FIGO grade } \\
\hline Low & 10 & 0 & 3 & 15 & 0 & \\
\hline Moderate & 3 & 0 & 0 & 3 & 0 & 3 \\
\hline High & 26 & 0 & 10 & 4 & 0 & 5 \\
\hline \multicolumn{7}{|l|}{ FIGO stage } \\
\hline I & 0 & 5 & 0 & 12 & 15 & 7 \\
\hline II & 2 & 0 & 4 & 9 & 0 & 0 \\
\hline III & 27 & 3 & 11 & 9 & 0 & 4 \\
\hline IV & 0 & 0 & 0 & 0 & 0 & 0 \\
\hline \multicolumn{7}{|c|}{ Lymph nodes } \\
\hline Positive & 19 & 2 & 3 & 0 & 0 & 1 \\
\hline Negative & 11 & 8 & 12 & 9 & 7 & 6 \\
\hline No. of cases & 30 & 10 & 15 & 30 & 15 & 15 \\
\hline
\end{tabular}

CCOC: clear cell ovarian carcinoma; EOC: endometrioid ovarian carcinoma; HG-PSC: high-grade papillary serous carcinoma; MMMT: malignant mixed Mullerian tumor; MOC: mucinous ovarian carcinoma; SBT: serous borderline tumor.

were prehybridized with $1 \times$ hybridization buffer without probe. The hybridization was carried out overnight in a $1 \times$ hybridization buffer $(30-70 \mu \mathrm{l})$ with predenatured miRCURY LNA, let-7b, let-7c or U6 probes. After washing, the slides were blocked and incubated with AP-conjugated anti-DIG Fab fragments (1:1500; Roche, Indianapolis, IN, USA) and visualized for color detection.

\section{Immunoscores, MicroRNA Scores and Statistical Analysis}

One-score system for immunointensity and microRNA intensity was used for the markers $H M G A 1$, HMGA2 and p53, and microRNA let-7b and let-7c. The semiquantitation for intensity was scored on a scale of 0 , negative; 1 , weak; 2 , moderate and 3 , strong. Another score system for percentage of immunopositivity was used for Ki-67 that was immunoreactive for only a portion of the tumor cells. The mean values and standard errors were calculated. Paired $t$-test was used. The $P$-values $<0.05$ were considered as statistically significant.

\section{Results}

\section{Expression of HMGA2 in Different Types of Ovarian Cancer}

In this study, we collected a total of 115 ovarian carcinomas from 6 different histological types, 
including high-grade papillary serous carcinoma, carcinosarcoma, serous borderline tumor, endometrioid ovarian carcinoma, mucinous ovarian carcinoma and clear cell ovarian carcinoma. In addition, sections from normal fallopian tubes and normal endometrium were collected as normal control. The detail biomedical factors of these tumor types are summarized in Table 1.

$H M G A 2$ was weakly and occasionally moderately expressed in ciliated cells of fallopian tube, but completely negative in secretory cells and endometrial stromal and epithelial cells (data not shown). ${ }^{20}$ In tumor sections, we defined HMGA2-positive tumors as those tumors with at least moderate immunoreactivity for HMGA2 (score $\geq 2$ ).

In high-grade papillary serous carcinomas, we found $64 \%$ of tumors (18 out of 28) were moderately to strongly immunoreactive for HMGA2 (Table 2). The immunopositive cells for HMGA2 ranged from 40 to $100 \%$. In contrast, only $10 \%$ serous borderline tumors (1 out of 10) were immunoreactive for HMGA2 (Table 2 and Figure 1).

In carcinosarcoma, the rate of immunoreactivity for HMGA2 was $60 \%$ of cases (9 out of 15) in carcinoma component and $47 \%$ (7 out of 15) in sarcoma (Table 2 and Figure 2). Most cases of carcinosarcoma contained carcinoma of serous differentiation. The similar rate of immunoreactivity for $H M G A 2$ in papillary serous carcinomas and carcinoma component of carcinosarcoma suggested a similar function of HMGA2 in these two types of ovarian malignancies. Among 30 cases of endometrioid ovarian carcinomas, only 2 cases $(7 \%)$ were immunoreactive for HMGA2. One of endometrioid ovarian carcinoma was strongly immunoreactive for both HMGA2 and p53 (Figure 1). Our findings indicated that endometrioid carcinoma of ovaries was mostly negative for HMGA2. About $23 \%$ of clear cell ovarian carcinomas ( 3 out of 13) were immunoreactive for HMGA2. Further study revealed that all three HMGA2-positive clear cell ovarian carcinomas had strong immunoreactivity for p53 (Figures 1 and 3). Of 15 mucinous ovarian carcinomas, 1 (6\%) was immunoreactive for HMGA2, which was the lowest rate of HMGA2 overexpression in all ovarian carcinomas (Table 2 and Figure 3).

Statistical analysis indicated that the rate and levels of immunoreactivity for HMGA2 were significantly higher in high-grade papillary serous carcinomas and carcinosarcomas than in other types of ovarian cancers $(P<0.05)$ (Table 2$)$.

\section{HMGA1 and p53 Expression in Different Types of Ovarian Cancer}

High-mobility-group A1 gene (HMGA1) is one of the gene family members of HMGA2. HMGA1 expression in ovarian cancer is largely unknown. We examined HMGA1 expression in the serial sections of the same population of ovarian cancer. As in Table 2 and Figure 1, HMGA1 is weakly and moderately immunoreactive for all types of ovarian cancer. The percentage of HMGA1-positive cases ranged from $82 \%$ for endometrioid carcinoma to $100 \%$ for clear cell carcinoma. The average levels of HMGA1 ranged from 0.91 to 1.43 (Table 2). The levels and rates of immunoreactivity for HMGA1

Table 2 Differential expression of the selected markers in ovarian cancer

\begin{tabular}{|c|c|c|c|c|c|}
\hline & \multirow[t]{2}{*}{ No. of cases } & \multicolumn{4}{|c|}{ Immunointensity (mean \pm s.e.m.) } \\
\hline & & $H M G A 1$ & HMGA2 & p53 & $K i-67$ \\
\hline HG-PSC & 30 & $1.06 \pm 0.11$ & $1.57 \pm 0.23$ & $1.79 \pm 0.26$ & $38.46 \pm 5.10$ \\
\hline MMMT & 15 & $1.43 \pm 0.13$ & $1.50 \pm 0.31$ & $2.00 \pm 0.35$ & $49.64 \pm 5.36$ \\
\hline SBT & 10 & $1.43 \pm 0.10$ & $0.22 \pm 0.21$ & $0.44 \pm 0.28$ & $16.25 \pm 5.81$ \\
\hline EOC & 30 & $0.91 \pm 0.09$ & $0.14 \pm 0.10$ & $0.75 \pm 0.23$ & $31.07 \pm 5.39$ \\
\hline MOC & 15 & $1.07 \pm 0.15$ & $0.13 \pm 0.13$ & $0.27 \pm 0.17$ & $18.34 \pm 5.67$ \\
\hline CCOC & 15 & $1.08 \pm 0.08$ & $0.54 \pm 0.27$ & $0.46 \pm 0.23$ & $27.69 \pm 6.69$ \\
\hline \multirow[t]{3}{*}{$P$-values } & $>0.05$ & $<0.05$ & $<0.05$ & $>0.05$ & \\
\hline & & \multicolumn{3}{|c|}{ No. of immunopositivity (\%) } & \\
\hline & & $H M G A 1$ & HMGA2 & $p 53^{\mathrm{a}}$ & \\
\hline HG-PSC & 30 & $24(85.7)$ & $18(64.3)$ & $18(64.3)$ & \\
\hline MMMT & 15 & $15(100)$ & $9(64.3)$ & $10(66.7)$ & \\
\hline SBT & 10 & $8(88.9)$ & $1(10.0)$ & $2(20.0)$ & \\
\hline EOC & 30 & $23(82.1)$ & $2(7.1)$ & $8(28.6)$ & \\
\hline MOC & 15 & $13(86.7)$ & $1(6.7)$ & $2(13.3)$ & \\
\hline CCOC & 15 & $15(100)$ & $3(23.1)$ & $3(23.1)$ & \\
\hline
\end{tabular}

CCOC: clear cell ovarian carcinoma; EOC: endometrioid ovarian carcinoma; HG-PSC: high-grade papillary serous carcinoma; MOC: mucinous ovarian carcinoma; MMMT: malignant mixed Mullerian tumor; SBT: serous borderline tumor.

${ }^{\mathrm{a}}$ Score $\geq 2$ was counted as immunopositive case. 

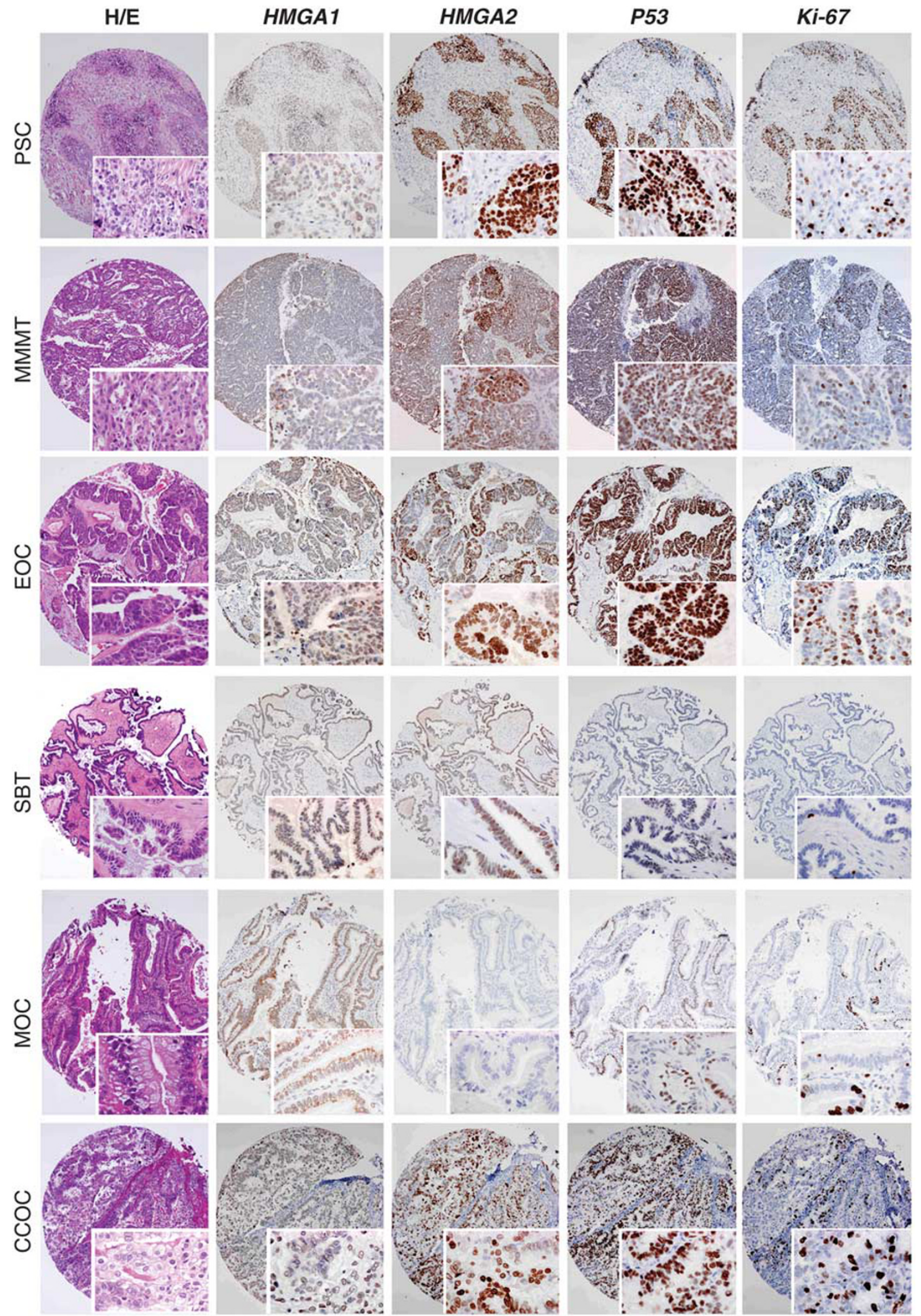

Figure 1 Photomicrographs illustrate examples of immunoreactivity for HMGA1, HMGA2, p53 and Ki-67 in six different histological types of ovarian cancer. Hematoxylin and eosin (H/E) and immunohistochemical stains for the selected markers were performed in serial sections of tissue core with low magnification $(\times 4)$ and inset with high magnification $(\times 40)$. PSC, high-grade serous papillary carcinoma; MMMT, carcinosarcoma; EOC, endometrioid ovarian carcinoma; SBT, serous borderline tumor; MOC, mucinous ovarian carcinoma; CCOC, clear cell ovarian carcinoma. 

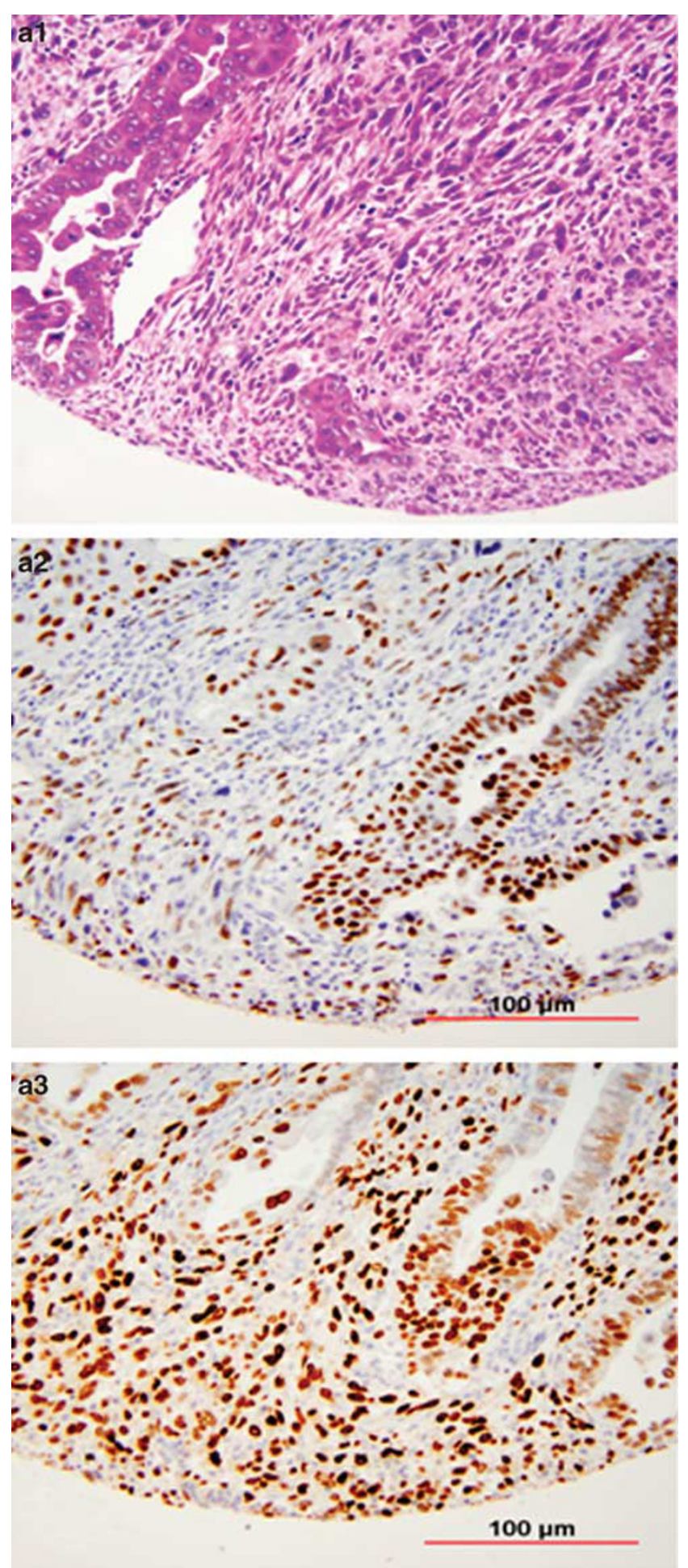

Figure 2 Photomicrographs illustrate immunoreactivity for HMGA2 (a2) and p53 (a3) in carcinosarcoma. Examples showed immunoreactivity for $H M G A 2$ and p53 were present in both epithelial and stromal components. (a1) H/E stain.

were statistically insignificant among different types of ovarian cancer $(P>0.05)$. We further examined the expression of p53 in the serial sections of all selected cases. We defined p53-positive cases as at
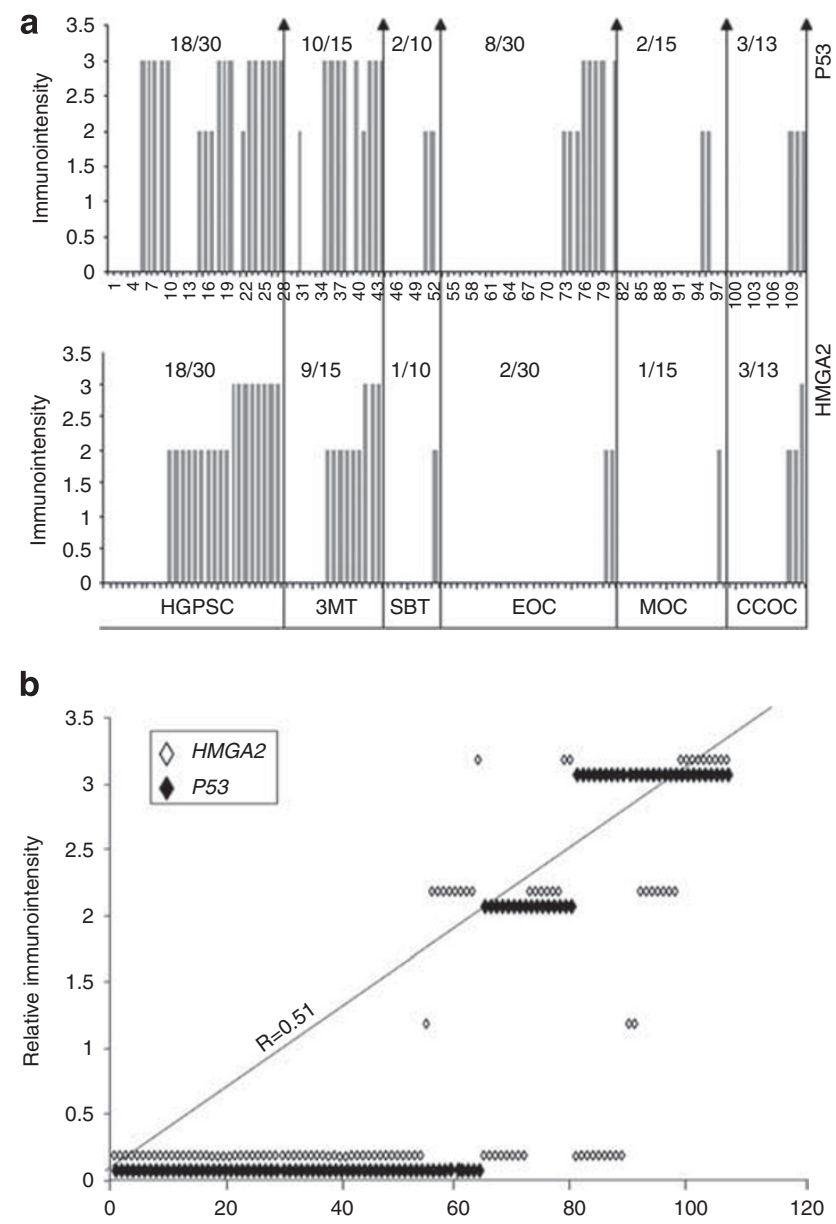

Figure 3 Immunoprofile analyses of $H M G A 2$ and p53 in six different histological types of ovarian cancer. (a) Histogram (each gray bar representing one case) shows the relative levels (y axis, immunointensity) and rates (above histobars) of HMGA2 (lower) and p53 (upper) expression in six different types of ovarian cancer. The relative expression was sorted based on the HMGA2 expression from negative to the highest levels. Arrow bars separate each tumor type (indicated at bottom). HGPSC, highgrade serous papillary carcinoma; MMMT, carcinosarcoma; EOC, endometrioid carcinoma; SBT, serous borderline tumor; MOC, mucinous ovarian carcinoma; CCOC, clear cell ovarian carcinoma. (b) Correlation analysis of HMGA2 (red dots) with p53 (blue dots) in 115 cases of ovarian cancer.

least moderate and diffuse immunoreactivity for p53. We found that the rate of immunoreactivity for p53 varied widely among different types of ovarian cancers. High-grade papillary serous carcinomas and carcinosarcomas had the highest rate of p53 immunopositivity, $64 \%$ and $67 \%$, respectively. All other types of ovarian cancers showed low rates of p53 immunoreactivity ranging from 13 to $28 \%$ of cases (Table 2 and Figure 3). Statistical analysis indicated that p53 was significantly higher in highgrade papillary serous carcinomas and carcinosarcomas than in other types of ovarian cancers (Table 2). In serial sections, we found that expression of p53 was moderately correlated with HMGA2 expression $(r=0.51)$ as shown in Figure $3 \mathrm{~b}$. 
The proliferation index (immunopositivity for Ki67 ) in six different types of ovarian cancers varied widely. Overall, carcinosarcoma had the highest $\mathrm{Ki}$ 67 index (50\% positive cells), followed by highgrade papillary serous carcinoma (39\%), endometrioid ovarian carcinoma (31\%) and clear cell ovarian carcinoma $(27 \%)$. Mucinous ovarian carcinoma and serous borderline tumor had the lowest index at 18 and $16 \%$, respectively. By correlation analysis, there were weak correlations between HMGA2 and p53, and HMGA2 and Ki-67 $(r=0.35$ and 0.21 , respectively). The weak correlation of HMGA2 with Ki-67 was negatively contributed by endometrioid ovarian carcinoma, in which, only lower rate of HMGA2 and higher rate of Ki-67 were observed (Table 2).

\section{let-7 Expression in High-Grade Papillary Serous Carcinomas and Endometrioid Ovarian Carcinomas}

let-7s were found to be downregulated in ovarian cancer. To characterize which types of ovarian cancer had significant downregulation of let-7s, we selected two let-7 family members, let-7b and let-7c for the study. In contrast to HMGA2 that is only expressed in tumor cells, but not in normal counterpart, let-7s are expressed in both normal and tumor cells. Therefore, to determine the differential examination of microRNAs let-7s, matched normal controls have to be used. As shown in Materials and methods section, in ovarian cancer of high-grade papillary serous carcinoma and endometrioid ovarian carcinoma, the case-matched normal tissue controls from fallopian tube and endometrium were collected. We acknowledged that normal endometrium and fallopian tube might not be the best controls for ovarian high-grade papillary serous carcinomas and endometrioid ovarian carcinomas, but they were the closest one we could get. We examined let-7b and let-7c expression by microRNA in situ hybridization, along with $U 6$ as internal small RNA control.

We found that there was significant downregulation of let-7b in high-grade papillary serous carcinomas $(1.04 \pm 0.14)$ in comparison to matched fallopian tube epithelium $(2.04 \pm 0.10) \quad(P<0.05)$ (Figure 4). Downregulation of let-7c had a similar pattern but less significant as let-7c had lower expression level in both normal fallopian tube epithelia $(1.31 \pm 0.11)$ and tumor cells $(0.69 \pm 0.13)$. No significant differences of let-7 expression were noted between endometrioid ovarian carcinomas (let-7b, $1.35 \pm 0.11$; let-7c, $0.77 \pm 0.16)$ and endometrium (let-7b, $1.45 \pm 0.11 ; \quad$ let-7c, $0.86 \pm 0.12$ ) (Figure 4).

\section{Discussion}

HMGA2 overexpression in ovarian cancer has been reported. Malek et $a l^{7}$ found that over $65 \%$ of ovarian serous carcinoma were positive for $H M G A 2$, whereas all normal ovarian epithelial lined tissues were negative for $H M G A 2 .^{7}$ Shell et al ${ }^{16}$ examined HMGA2 expression by immunohistochemistry in 100 primary ovarian cancer tissues $(77 \%$ were serous type) and they found that high expression of HMGA2 significantly correlated with an adverse prognosis.

Ovarian cancer is a group of heterogeneous diseases, involving different molecular pathways. To study the function of HMGA2 in ovarian cancer, it is important to define which types of ovarian cancer are associated with HMGA2 overexpression. Kurman and Shih ${ }^{21}$ proposed a model for ovarian tumorigenesis. Ovarian tumors are divided into two groups designated as type I and type II. Type I tumors are slow growing, generally confined to the ovary at diagnosis and develop from well-established precursor lesions. Type I tumors include lowgrade micropapillary serous, mucinous, endometrioid and clear cell carcinomas. They are genetically stable and are characterized by mutations in a number of different genes, including KRAS, BRAF, PTEN and $\beta$-catenin. ${ }^{3,22,23}$

Type II tumors are rapidly growing highly aggressive neoplasms for which precursor lesions arising from Fimbria are currently under intensive studies. $^{24}$ Type II tumors include high-grade serous carcinoma, carcinosarcoma and undifferentiated carcinoma. This group of tumors has a high level of genetic instability and is characterized by mutations of $p 53 .^{3}$ In this study, we found HMGA2 overexpression was commonly seen in type II tumors, including high-grade papillary serous carcinoma and carcinosarcoma. In addition, we found that HMGA2 overexpression is moderately correlated with p53 expression (Figure 3). We propose that HMGA2 overexpression is an important biomarker, in conjugated with p53, for the diagnosis and possibly associated with tumorigenesis of type II ovarian carcinoma.

In a recent study, we found that serous intraepithelial carcinoma in fallopian tube had high rate and level of HMGA2 overexpression along with p53-dominant mutations. ${ }^{20}$ The findings indicate that HMGA2 overexpression is an early event in the tumorigenesis of high-grade papillary serous carcinoma. Further study by characterization of functional relationship between p53 mutations and HMGA2 overexpression will be valuable for understanding the functional task of HMGA2 in the tumorigenesis of high-grade papillary serous carcinoma.

HMGA2 overexpression is present in many different epithelial and mesenchymal neoplasms. HMGA2 in regulation of the molecular pathways responsible for tumorigenesis and tumor progression were only partially understood. In the study of 60 cancer cell lines, Peter's research group found that HMGA2 is one of a few gene markers that can distinguish most type I (express mesenchymal gene signature) from 

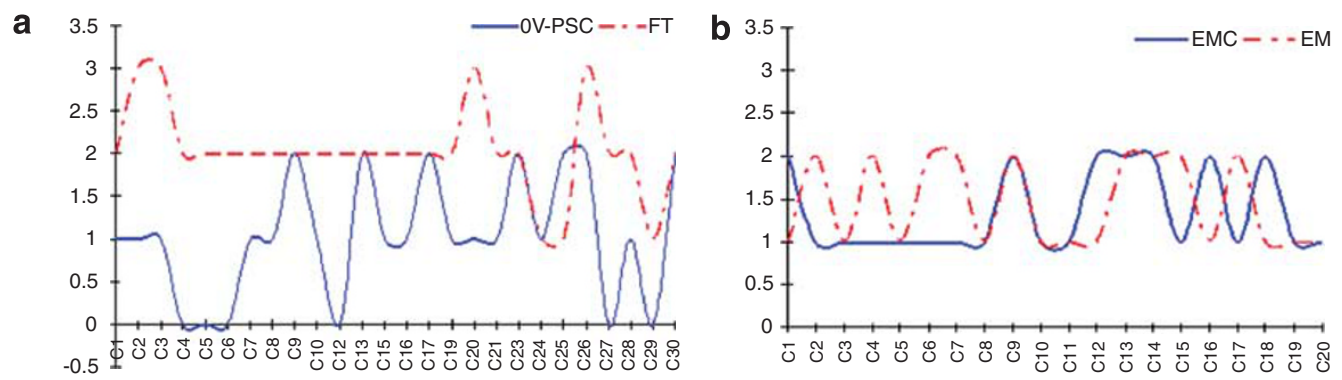

c
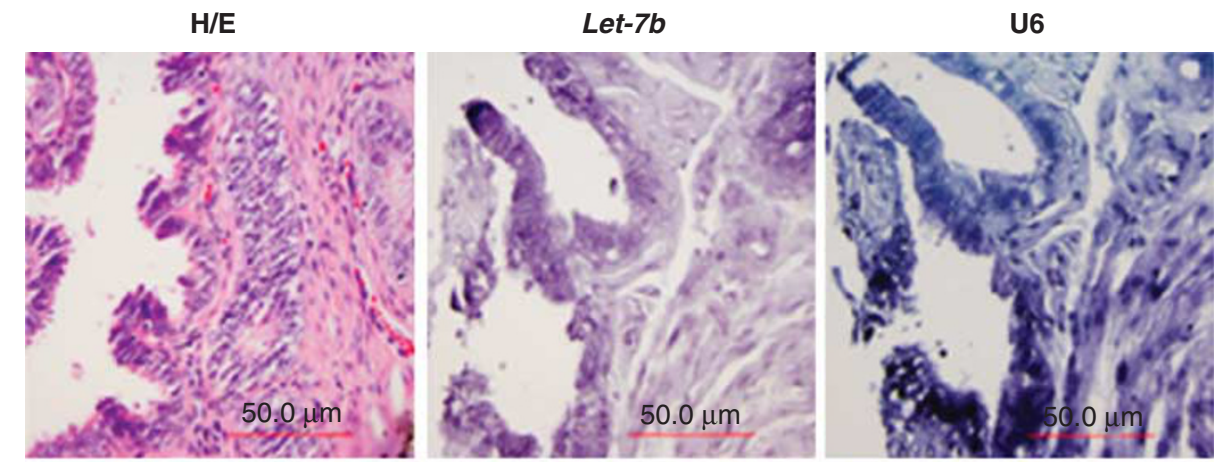

这
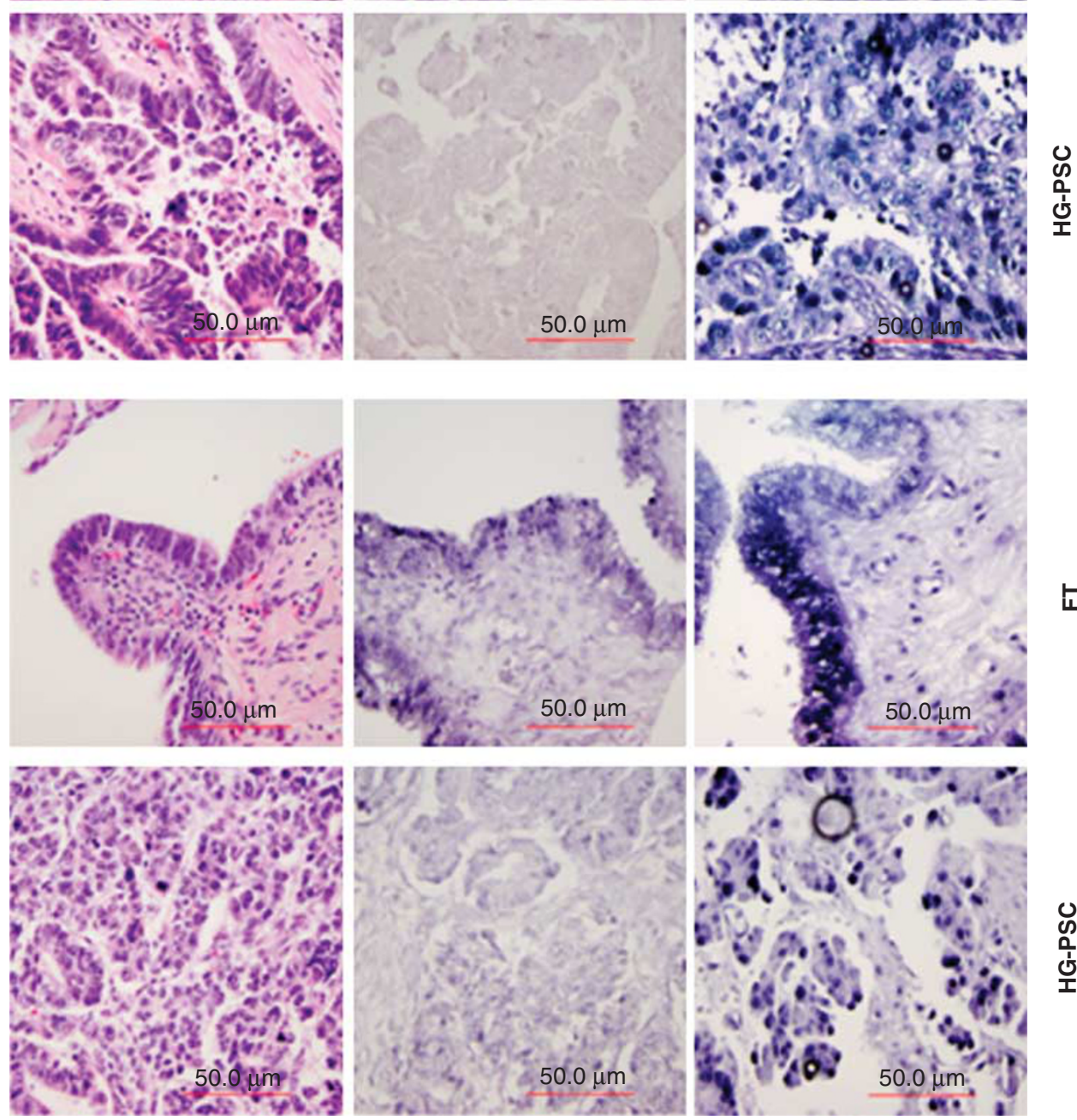

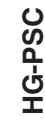

Figure 4 let-7b expression in high-grade papillary serous carcinomas (HG-PSC) and endometrioid ovarian carcinomas (EOC). (a) Relative expression of let-7b (y axis) in high-grade papillary serous carcinomas (red dot line) and matched fallopian tube epithelia (FT, blue solid line) in a total 30 cases. (b) Relative expression of let-7b (y axis) in endometrioid ovarian carcinomas (red dot line) and matched endometrium (EM, blue solid line) in a total 20 cases. (c) Photomicrographs illustrate some examples of intensity of let-7b expression in high-grade papillary serous carcinomas and FT. U6 is RNA quality control. The intensity of blue color indicates the levels of let-7b expression. 
type II (express epithelial gene signature) cancer cell lines. ${ }^{9,16}$ HMGA2 participates in epithelialmesenchymal transition, ${ }^{25,26}$ a key step in tumor plasticity by converting adherent epithelial cells to motile mesenchymal cells ${ }^{27}$ through the functional loss of E-cadherin, which is required to maintain epithelial cell-cell adhesion. ${ }^{27}$ HMGA2 overexpression is associated with tumor growth, ${ }^{28}$ differentiation, ${ }^{9,16,28}$ metastasis, ${ }^{29}$ unfavorable outcome and resistance to treatment. ${ }^{25,30}$ The function of HMGA2 in behaviors of aggressive tumor growth in ovarian high-grade papillary serous carcinomas deserves further investigation.

Members of the let-7 microRNA family function as tumor suppressors through specific repression of its target gene, particularly of HMGA2 expression in some tumor cells both in vivo and in vitro. ${ }^{17}$ The biological importance of molecular pairing of let7::HMGA2 has been shown by repression of HMGA2 by let-7s which impairs tumor cell proliferation in many different tumor types. ${ }^{14,15,17}$ To study whether overexpression of HMGA2 is associated with loss of let-7 expression, in this study, we examined two family members of let-7. We found that let-7b was significantly downregulated in high-grade papillary serous carcinoma against fallopian tube epithelia (Figure 4). We proposed that loss of let-7s can be part of pathogenesis in high-grade papillary serous carcinoma.

In conclusion, our study suggests that $H M G A 2$ overexpression is an important molecular change specifically related to high-grade papillary serous carcinomas. HMGA2 overexpression is associated with p53-dominant mutations and let-7 downregulation. In addition, $H M G A 2$ overexpression can be a potential biomarker in conjugation with $p 53$ for the detection and differentiation of ovarian high-grade papillary serous carcinomas.

\section{Acknowledgements}

We are grateful to Dr E Hernando who provided us with HMGA1 antibody. We thank Tongsheng Wang for data preparation. This study was supported by Dixon translation research grant. A part of this work was presented in 97th United States and Canadian Academy of Pathology in Boston 2008.

\section{Disclosure/conflict of interest}

The authors declare no conflict of interest.

\section{References}

1 American Cancer Society. Cancer Facts and Figures 200. American Cancer Society: Atlanta, 2008.

2 Kobel M, Kalloger SE, Boyd N, et al. Ovarian carcinoma subtypes are different diseases: implications for biomarker studies. PLoS Med 2008;5:e232.
3 Veras E, Mao TL, Ayhan A, et al. Cystic and adenofibromatous clear cell carcinomas of the ovary: distinctive tumors that differ in their pathogenesis and behavior: a clinicopathologic analysis of 122 cases. Am J Surg Pathol 2009;33:844-853.

4 Gattas GJ, Quade BJ, Nowak RA, et al. HMGIC expression in human adult and fetal tissues and in uterine leiomyomata. Genes Chromosomes Cancer 1999;25:316-322.

5 Rogalla P, Drechsler K, Frey G, et al. HMGI-C expression patterns in human tissues. Implications for the genesis of frequent mesenchymal tumors. Am J Pathol 1996;149:775-779.

6 Reeves R. Structure and function of the HMGI(Y) family of architectural transcription factors. Environ Health Perspect 2000;108(Suppl 5):803-809.

7 Malek A, Bakhidze E, Noske A, et al. HMGA2 gene is a promising target for ovarian cancer silencing therapy. Int J Cancer 2008;123:348-356.

8 Malek AV, Bakhidze EV. [Role of genome research in the diagnosis and therapy of ovarian cancer]. Vopr Onkol 2005;51:182-186.

9 Park SM, Shell S, Radjabi AR, et al. Let-7 prevents early cancer progression by suppressing expression of the embryonic gene HMGA2. Cell Cycle 2007;6: 2585-2590.

10 Welsh JB, Zarrinkar PP, Sapinoso LM, et al. Analysis of gene expression profiles in normal and neoplastic ovarian tissue samples identifies candidate molecular markers of epithelial ovarian cancer. Proc Natl Acad Sci USA 2001;98:1176-1181.

11 Berlingieri MT, Manfioletti G, Santoro M, et al. Inhibition of HMGI-C protein synthesis suppresses retrovirally induced neoplastic transformation of rat thyroid cells. Mol Cell Biol 1995;15:1545-1553.

12 Pentimalli F, Dentice M, Fedele M, et al. Suppression of HMGA2 protein synthesis could be a tool for the therapy of well differentiated liposarcomas overexpressing HMGA2. Cancer Res 2003;63:74237427.

13 Lee YS, Dutta A. The tumor suppressor microRNA let7 represses the HMGA2 oncogene. Genes Dev 2007;21: 1025-1030.

14 Mayr C, Hemann MT, Bartel DP. Disrupting the pairing between let-7 and Hmga2 enhances oncogenic transformation. Science 2007;315:1576-1579.

15 Pengetnze Y, Steed M, Roby KF, et al. Src tyrosine kinase promotes survival and resistance to chemotherapeutics in a mouse ovarian cancer cell line. Biochem Biophys Res Commun 2003;309:377-383.

16 Shell S, Park SM, Radjabi AR, et al. Let-7 expression defines two differentiation stages of cancer. Proc Natl Acad Sci USA 2007;104:11400-11405.

17 Wang $\mathrm{T}$, Zhang $\mathrm{X}$, Obijuru L, et al. A micro-RNA signature associated with race, tumor size, and target gene activity in human uterine leiomyomas. Genes Chromosomes Cancer 2007;46:336-347.

18 Zhao XW, Li Y, Wang N, et al. [Study on the association of SNPs of MMP-2 and TIMP-2 genes with the risk of endometriosis and adenomyosis]. Zhonghua Yi Xue Yi Chuan Xue Za Zhi 2008;25:280-283.

19 Kloosterman WP, Wienholds E, Ketting RF, et al. Substrate requirements for let-7 function in the developing zebrafish embryo. Nucleic Acids Res 2004;32:6284-6291.

20 Wei J, Wu J, Luan C, et al. HMGA2: a potential biomarker complement to P53 for detection of 
early-stage high-grade papillary serous carcinoma in fallopian tubes. Am J Surg Pathol 2010;34:18-26.

21 Kurman RJ, Shih IeM. Pathogenesis of ovarian cancer: lessons from morphology and molecular biology and their clinical implications. Int J Gynecol Pathol 2008;27:151-160.

22 Geyer JT, Lopez-Garcia MA, Sanchez-Estevez C, et al. Pathogenetic pathways in ovarian endometrioid adenocarcinoma: a molecular study of 29 cases. Am J Surg Pathol 2009;33:1157-1163.

23 Obata K, Hoshiai H. Common genetic changes between endometriosis and ovarian cancer. Gynecol Obstet Invest 2000;50(Suppl 1):39-43.

24 Rohen C, Rogalla P, Meyer-Bolte K, et al. Pleomorphic adenomas of the salivary glands: absence of HMGIY rearrangements. Cancer Genet Cytogenet 1999;111: 178-181.

25 Watanabe S, Ueda Y, Akaboshi S, et al. HMGA2 maintains oncogenic RAS-induced epithelial-mesenchymal transition in human pancreatic cancer cells. Am J Pathol 2009;174:854-868.

26 Thuault S, Tan EJ, Peinado $\mathrm{H}$, et al. HMGA2 and Smads co-regulate SNAIL1 expression during induction of epithelial-to-mesenchymal transition. J Biol Chem 2008;283:33437-33446.

27 Polyak K, Weinberg RA. Transitions between epithelial and mesenchymal states: acquisition of malignant and stem cell traits. Nat Rev Cancer 2009;9:265-273.

28 Peng Y, Laser J, Shi G, et al. Antiproliferative effects by Let-7 repression of high-mobility group A2 in uterine leiomyoma. Mol Cancer Res 2008;6: 663-673.

29 Hristov AC, Cope L, Reyes MD, et al. HMGA2 protein expression correlates with lymph node metastasis and increased tumor grade in pancreatic ductal adenocarcinoma. Mod Pathol 2009;22:43-49.

30 Fusco A, Fedele M. Roles of HMGA proteins in cancer. Nat Rev Cancer 2007;7:899-910. 